\title{
High speed partial Stokes imaging using a ferroelectric liquid crystal modulator
}

\author{
Albéric Jaulin \\ Alberic.Jaulin@uha.fr \\ Laurent Bigué \\ Laurent.Bigue@uha.fr
}

\begin{abstract}
Laboratoire Modélisation Intelligence Processus Systèmes (EA 2332), École Nationale Supérieure d’Ingénieurs Sud Alsace, Université de Haute-Alsace, 12, rue des Frères Lumière - 68093 Mulhouse Cedex - France

Laboratoire Modélisation Intelligence Processus Systèmes (EA 2332), École Nationale Supérieure d'Ingénieurs Sud Alsace, Université de Haute-Alsace, 12, rue des Frères Lumière - 68093 Mulhouse Cedex - France
\end{abstract}

This paper describes the implementation of an imaging polarimeter using a single ferroelectric liquid crystal light modulator. It provides partial imaging Stokes information at $234 \mathrm{~Hz}$, much faster than polarimeters using nematic light modulators. This information is obtained for dynamic scenes in reflection or transmission. Partial Stokes information contains the first three Stokes parameters. With this information, imaging of the linear degree of polarization and of the angle of polarization can be obtained and imaging linear depolarization can be quantified. [DOI: 10.2971/jeos.2008.08019]

Keywords: polarimetry, imaging system, Stokes vector, light modulator, ferroelectric liquid crystal device

\section{INTRODUCTION}

Polarization imaging can provide information which does not appear in classic intensity imaging. Applications of imaging polarimetry can be found in a growing number of fields: medical imaging [1, 2], metrology [3]-[5], remote sensing [6, 7] or for the discrimination between materials with the same appearance [8]. In some cases, partial polarimetric information may be sufficient: circularly polarized light is not useful and only linearly polarized light is considered, which comes down to evaluating the first three Stokes parameters. 3D reconstruction, in a general case [9] or in the case of metallic surfaces [10], determination of rotatory power [11] or atmospheric studies [12] are a few examples among many other ones.

Imaging polarimetric information is often evaluated for static scenes, since the devices used to produce polarization images usually operate at low frame rates, below $30 \mathrm{~Hz}$. Polarization imaging of dynamic scenes definitely requires much faster devices. Several implementations of dynamic imaging polarimeters have been proposed over the last few years [13]. Some of them use amplitude division, at the expense of a complicated optical setup, or wavefront division, at the expense of a lower image resolution [14]. Like many other authors, we implement a sequential polarization information production. Getting all possible information about linear polarization, ie degree of polarization and direction, comes down to evaluating the first three Stokes parameters. Such implementations have already been proposed, using either two bistable modulators $[15,16]$ or a single accordable modulator. Fast bistable modulators such as ferroelectric liquid crystal (FLC) devices are widespread. Accordable modulators are usually nematic liquid crystal light modulators, but the latter usually run at low frame rates, typically $20-50 \mathrm{~Hz}$. In this paper, we propose to use an accordable high speed ferroelectric light modulator as a polarization rotator in an imaging polarimeter.

Section 2 reviews the partial Stokes vector evaluation and the linear degree of polarization. Section 3 presents our polarimeter implemented with a FLC modulator and its Mueller characterization. In Section 4, we present some results for a reflective or a transmissive scene. Section 5 discusses our implementations and results and highlights possible improvements.

\section{PARTIAL STOKES VECTOR EVALUATION}

\subsection{Definition of Stokes vector}

Stokes formalism fully describes the polarization state of a light wave with four parameters named Stokes vector which can be derived from $2 \times 2$ coherence matrix J [13] .

$$
\left(\begin{array}{l}
S_{0} \\
S_{1} \\
S_{2} \\
S_{3}
\end{array}\right)=\left(\begin{array}{c}
J_{x x}+J_{y y} \\
J_{x x}-J_{y y} \\
J_{x y}+J_{y x} \\
i\left(J_{x y}-J_{y x}\right)
\end{array}\right)
$$

The first parameter $S_{0}$ describes the total light intensity. Information about polarization is contained in the other three parameters $S_{1}, S_{2}$ and $S_{3}$. $S_{1}$ describes the horizontal and vertical polarizations, $S_{2}$ the components at $\pm 45^{\circ}$ and $S_{3}$ the right and left circular polarizations. The transfer matrix between two Stokes vectors is named Mueller matrix. This matrix describes polarization behaviour of light through a material and 
its coefficients are named as:

$$
M=\left[\begin{array}{llll}
m_{11} & m_{12} & m_{13} & m_{14} \\
m_{21} & m_{21} & m_{23} & m_{24} \\
m_{31} & m_{31} & m_{33} & m_{34} \\
m_{41} & m_{41} & m_{43} & m_{44}
\end{array}\right]
$$

The following relations between all parameters of Stokes vector can be established [13]:

$$
\begin{gathered}
S_{0}^{2} \geq S_{1}^{2}+S_{2}^{2}+S_{3}^{2} \\
D O P=\frac{\sqrt{S_{1}^{2}+S_{2}^{2}+S_{3}^{2}}}{S_{0}}
\end{gathered}
$$

If $S_{1}, S_{2}$ and $S_{3}$ are zero, the beam is totally unpolarized and the Degree Of Polarization $(D O P)$ is zero. In most cases, the beam is partially polarized, therefore the inequality (3) is strict and $D O P$ is smaller than one. In the case of a fully polarized beam, (3) tends to a strict equality and DOP is equal to unity.

When studying linear polarization, $S_{3}$ is not considered or considered to be nil. In this case, the Stokes vector is partial and is defined as:

$$
S=\left(\begin{array}{c}
S_{0} \\
S_{1} \\
S_{2} \\
-
\end{array}\right)
$$

Therefore, the degree of polarization becomes the linear degree of polarization $D O P_{L}$ defined by:

$$
\operatorname{DOP}_{L}=\frac{\sqrt{S_{1}^{2}+S_{2}^{2}}}{S_{0}}
$$

The value of $D O P_{L}$ is between zero and one. $D O P_{L}$ permits evaluation of depolarization [17], distinction between materials like metallic or scattering surfaces $[18,19]$, painted surfaces [20] or some most natural or man-made objects [5]. It should be noticed that $D O P_{L}$ is of course smaller than $D O P$ and not considering $S_{3}$ comes down to consider circular polarization like an unpolarized component.

A further parameter, the polarization angle ( $\Psi$ ) can be evaluated with the partial Stokes vector [13]:

$$
\tan (2 \Psi)=\frac{S_{2}}{S_{1}}
$$

The polarization angle allows the user to evaluate the orientation of linear polarization.

\subsection{Calculation of imaging partial Stokes vector}

\subsubsection{Principle and static implementations}

Static methods for the imaging evaluation of partial Stokes vector classically use as a Polarization State Analyzer (PSA) a rotating polarizer or a rotating half-wave plate before a fixed polarizer. Both setups correspond to different Mueller matrices. With a rotating half-wave plate in front of a fixed vertical polarizer, which will be considered in the following, the overall Mueller matrix depends on the orientation difference between the wave plate and the polarizer (Eq. 8), resulting from the combination of the respective Mueller matrices of the polarizer $M_{p o l}$ and of the half-wave plate $M_{\frac{\lambda}{2}}$ :

$M_{P S A(\theta)}=M_{p o l\left(0^{\circ}\right)} \cdot M_{\frac{\lambda}{2}(\theta)}=\frac{1}{2}\left[\begin{array}{cccc}1 & \cos (4 \theta) & \sin (4 \theta) & 0 \\ 1 & \cos (4 \theta) & \sin (4 \theta) & 0 \\ 0 & 0 & 0 & 0 \\ 0 & 0 & 0 & 0\end{array}\right]$

Let us consider various configurations (ie various angles $\theta_{i}$ ) for the polarimetric setup, leading to various output Stokes vectors $S_{i}^{\prime}$. All the output information is contained in the first Stokes component $S_{i, 0}^{\prime}$, which can be measured as the intensity $I_{i}$. The intensities $I_{i}$ can be gathered into a single vector $I$, and the analysis matrix $A$ [21], characterizing the capacity of partial Stokes evaluation, can be defined as:

$$
S_{i}^{\prime}=\left[\begin{array}{c}
S_{i, 0}^{\prime} \\
S_{i, 1}^{\prime} \\
S_{i, 2}^{\prime} \\
S_{i, 3}^{\prime}
\end{array}\right]=\left[\begin{array}{c}
I_{\theta_{i}} \\
I_{\theta_{i}} \\
0 \\
0
\end{array}\right]=M_{P S A\left(\theta_{i}\right)} \cdot\left[\begin{array}{c}
S_{0} \\
S_{1} \\
S_{2} \\
S_{3}
\end{array}\right]
$$

$$
I=\left[\begin{array}{c}
I_{1} \\
I_{2} \\
\vdots \\
I_{n}
\end{array}\right]=A . S=A\left[\begin{array}{c}
S_{0} \\
S_{1} \\
S_{2} \\
S_{3}
\end{array}\right]
$$

where $S$ is the unknown Stokes vector. Various values of $\theta$ are considered, leading to the following expression of the analysis matrix:

$$
A=\frac{1}{2}\left[\begin{array}{cccc}
1 & \cos \left(4 \theta_{1}\right) & \sin \left(4 \theta_{1}\right) & 0 \\
1 & \cos \left(4 \theta_{2}\right) & \sin \left(4 \theta_{2}\right) & 0 \\
\vdots & \vdots & \vdots & \vdots \\
1 & \cos \left(4 \theta_{n}\right) & \sin \left(4 \theta_{n}\right) & 0
\end{array}\right]
$$

Since the rank of the analysis matrix $A$ is 3 or less, we cannot gain access to the full Stokes vector which requires a 4rank matrix. To do so, an element, like a quarter wave plate, transforming at least partially linear polarization into circular polarization is required.

Finally, we can evaluate the unknown incident Stokes vector by performing a pseudo-inverse calculus: 


$$
\left[\begin{array}{l}
S_{0} \\
S_{1} \\
S_{2}
\end{array}\right]=A_{r}^{+} \cdot I=\left(A_{r}^{t} \cdot A_{r}\right)^{-1} \cdot A_{r}^{t} \cdot I
$$

where

$$
A_{r}=\frac{1}{2}\left[\begin{array}{ccc}
1 & \cos \left(4 \theta_{1}\right) & \sin \left(4 \theta_{1}\right) \\
1 & \cos \left(4 \theta_{2}\right) & \sin \left(4 \theta_{2}\right) \\
\vdots & \vdots & \vdots \\
1 & \cos \left(4 \theta_{n}\right) & \sin \left(4 \theta_{n}\right)
\end{array}\right]
$$

\subsubsection{Dynamic implementations}

Choosing a liquid crystal modulator to determine a Stokes vector is interesting when dynamic scenes are considered. Historically, the first method used two twisted nematic liquid crystal modulators used with binary controls. This method developed by Wolff et al.[15] allows the user to gain access to the first three Stokes parameters, since it consists of a modified version of one of the setups described in the previous Section: modulators used in binary mode act as switchable half wave plates. They are chosen with different rotary powers, so that the setup produces four different directions of analysis. Typical switching time for these modulators is $30 \mathrm{~Hz}$, too slow to reach the classical $25 \mathrm{~Hz}$ or $30 \mathrm{~Hz}$ video frame rate.

To evaluate the last Stokes parameter $S_{3}$, a single parallel aligned nematic liquid crystal modulator can be used, at a maximum frequency of $30 \mathrm{~Hz}[22,23]$. This modulator behaves as a variable retardation plate. This method gives all Stokes parameters, but remains slow.

A way to increase acquisition frame rate consists in using two bistable ferroelectric liquid crystal (FLC) modulators, but it can only evaluate the first three Stokes parameters. A first implementation uses two identical modulators and two fixed retardation plates. In this setup, FLC modulators behave as bistable half wave plates [24]. Another implementation consists in using a combination of two bistable FLC modulators with respective retardation of $\lambda / 2$ and of $3 \lambda / 4$ [16]. We aim at developing a technique which requires only a single high speed modulator.

\section{PARTIAL STOKESMETER WITH FERROELECTRIC LIQUID CRYSTAL MODULATOR}

We propose to use a single non-bistable FLC modulator with a standard CCD high speed camera in order to implement a partial Stokes polarimeter.

\subsection{Features}

The two liquid crystal modulators of setups inspired by Wolff's dynamic systems described in Section 2.2.2 are replaced by a single ferroelectric liquid crystal reflective modulator capable of reaching up to $1 \mathrm{kHz}$ [25].

This modulator, manufactured by BNS Inc., is supposed to act as a half-wave plate whose axis is continuously tunable from $0^{\circ}$ to $45^{\circ}$. Unfortunately, this modulator is a half-wave plate only for its design wavelength $[25,26]$. Ferroelectric liquid crystal cells can be operated at several tens of $\mathrm{kHz}$, but this device is pixelated $(512 \times 512$ pixels $)$ and the electronic addressing scheme limits its frame rate to $1015 \mathrm{~Hz}$ [27, 28]. It has a 15-micrometer pitch and each individual pixel can be controlled with eight bits. This device exhibits a response versus grey level which is varying according to the frame rate [29]. It should also be noticed that FLC cells require to get a zero time-average voltage, and then, for each image sent to the modulator, the hardware driver sends the inverse image. Consequently, the usable view time is divided by 2 .

This device was already used to evaluate partial linear DOP. In this previous configuration, only two controls corresponding to grey levels 0 and 255 were required to drive the modulator [30]. Partial Stokes vector requires an additional control in order to get an intermediate polarization rotation, but due to the modulator nonlinear response, the corresponding grey level has been determined thanks to a full Mueller matrix characterization.

\section{2 optical characterization}

The full optical setup (exit polarizer apart) has been characterized with Mueller formalism (Figure 1). A previous characterization [29] showed that the FLC device behaviour was rather dependent on the frame rate. Since the purpose of this paper is to demonstrate, as a first validation, the point in using a tunable FLC modulator as a PSA, changes due to operating in a wide range of frame rates were not investigated. The study was carried out at $234 \mathrm{~Hz}$ frame rate and a wavelength of 632.8 $\mathrm{nm}$. A rotating diffuser, leading to $2 \%$ depolarization, was placed after the beam expander in order to remove speckle. Mueller matrix was evaluated for all possible control grey levels of the modulator. The Polarization State Generator (PSG)

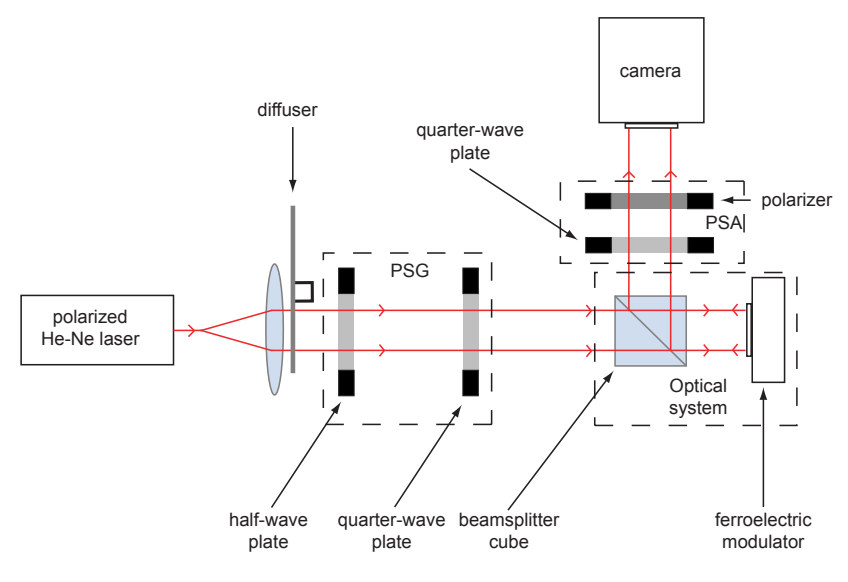

FIG. 1 Setup for Mueller characterization of the FLC device.

consists of a rotating quarter-wave plate before a rotating halfwave plate and the Polarization State Analyzer (PSA) consists of a rotating quarter-wave plate in front of a fixed vertical polarizer. The various 4 configurations of the PSG and their corresponding Stokes vector are listed in Table 1 . We used 5 positions for the PSA, namely $\left\{-\frac{\pi}{4},-\frac{\pi}{8}, 0, \frac{\pi}{8}, \frac{\pi}{4}\right\}$. Therefore, 


\begin{tabular}{|c|c|}
\hline $\begin{array}{c}\text { Configurations (quarter-wave plate, } \\
\text { half-wave plate) }\end{array}$ & Stokes vector \\
\hline$(0,0)$ & $(1 ; 1 ; 0 ; 0)$ \\
\hline$\left(0, \frac{\pi}{2}\right)$ & $(1 ;-1 ; 0 ; 0)$ \\
\hline$\left(0, \frac{\pi}{4}\right)$ & $(1 ; 0 ; 1 ; 0)$ \\
\hline$\left(\frac{\pi}{4}, 0\right)$ & $(1 ; 0 ; 0 ; 1)$ \\
\hline
\end{tabular}

TABLE 1 Configurations of the PSG and their corresponding Stokes vector.

the characterization of each gray level requires $4 \times 5$ measurements.

The Mueller characterization of the overall setup is reported Figure 2. It clearly differs from that of a half-wave plate. A severe issue lies for instance in the fact that the experimental $m_{23}$ is close to the theoretical $m_{24}$ (zero), and reciprocally. It implies that linear $\pm 45^{\circ}$ components cannot be analyzed, whereas circular components can.
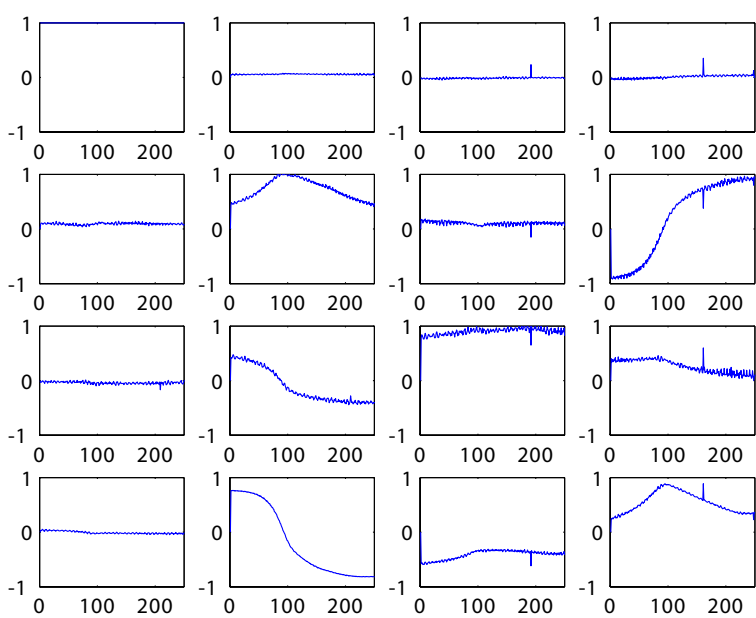

FIC. 2 The overall setup Mueller matrix. Each Mueller matrix coefficient is plotted versus grey level control of the modulator.

In order to better understand this phenomenon, the beamsplitter cube behaviour was separately investigated. It consists in studying both forward (normal entrance, Figure 3a) and sideward directions (opposite entrance, Figure 3b).

Mueller matrices for both directions are respectively named $M_{d}$ Eq. (14) and $M_{r}$ Eq. (15). These matrices notably differ from the identity matrix. They respectively correspond to the Mueller Matrices of a $70^{\circ}$ retardation plate $\left(M_{d}\right.$, forward direction) and to that of a $85^{\circ}$ retardation plate $\left(M_{r}\right.$, sideward direction).

$$
\begin{aligned}
& M_{d}=\left[\begin{array}{cccc}
1 & -0.018 & 0.002 & 0.016 \\
0.010 & 0.992 & -0.069 & 0.143 \\
0.008 & -0.065 & 0.369 & 0.888 \\
0.004 & -0.099 & -0.909 & 0.339
\end{array}\right] \\
& M_{r}=\left[\begin{array}{cccc}
1 & 0.005 & 0.041 & 0.036 \\
0.042 & 1.027 & -0.135 & -0.063 \\
0.020 & -0.100 & 0.056 & -1.021 \\
0.000 & 0.088 & 1.009 & 0.071
\end{array}\right]
\end{aligned}
$$
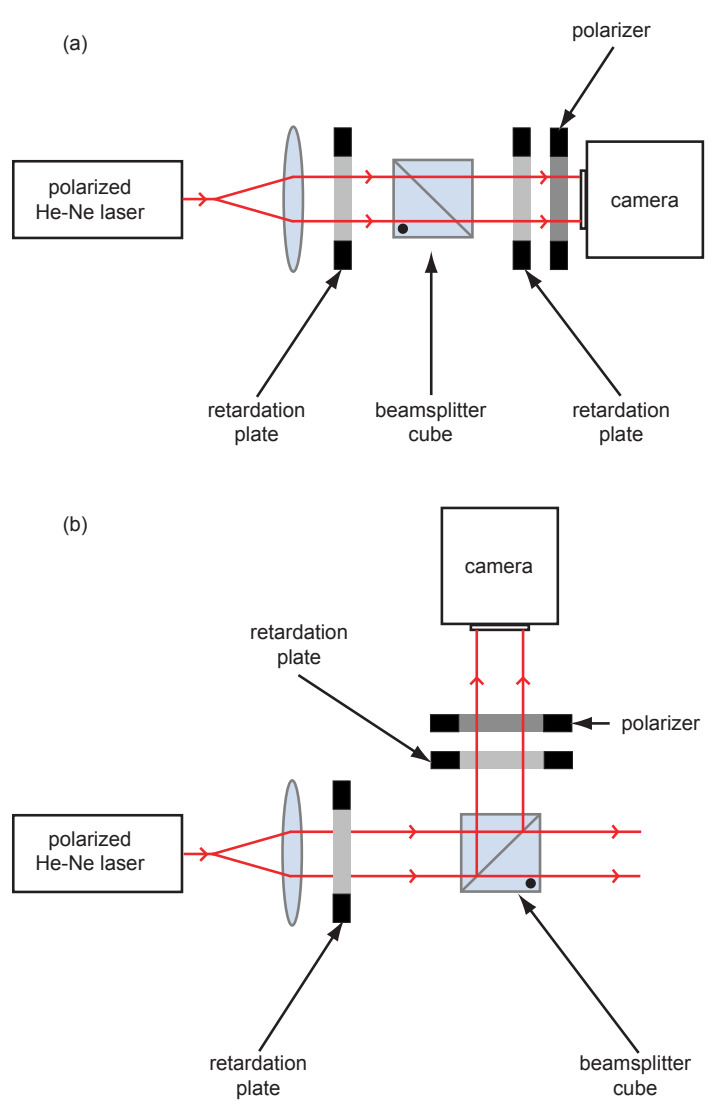

FIC. 3 Optical setups for the characterization of the beamsplitter cube. (a) Forward direction with normal entrance. (b) Sideward direction with opposite entrance.

The beamsplitter contribution can be numerically removed in order to get the behavior of the only FLC device (Figure 4).

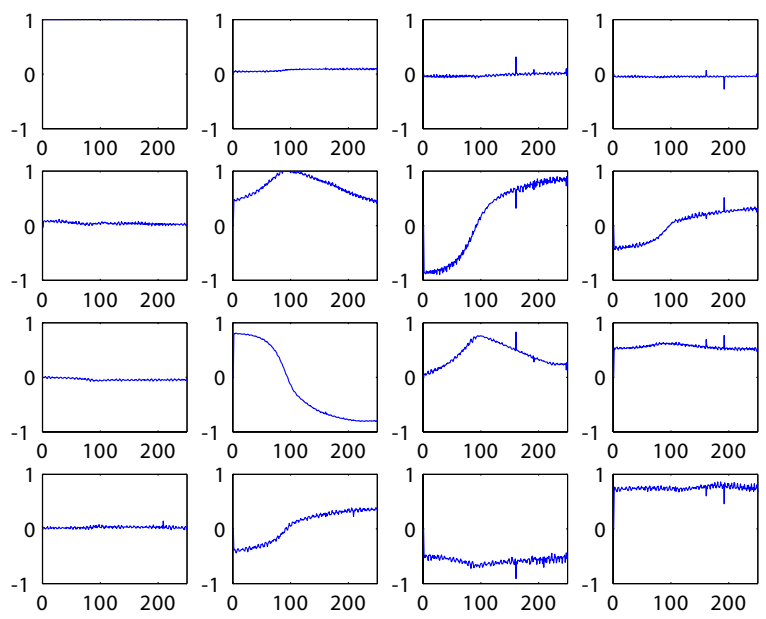

FIG. 4 FLC modulator Mueller matrix. Each Mueller matrix coefficient is plotted versus grey level control of the modulator. This chacterization only deals with the FLC device, the beamsplitter cube contribution was removed.

Up to a certain extent, the FLC device can be compared to a half-wave plate approximately rotating from -20 to $20^{\circ}$ (Figure 5), provided that its backplane mirror is taken into account (which for instance changes signs of the coefficients in the last two rows of the Mueller matrix).

As mentioned earlier, the beamsplitter cube, acting as a re- 


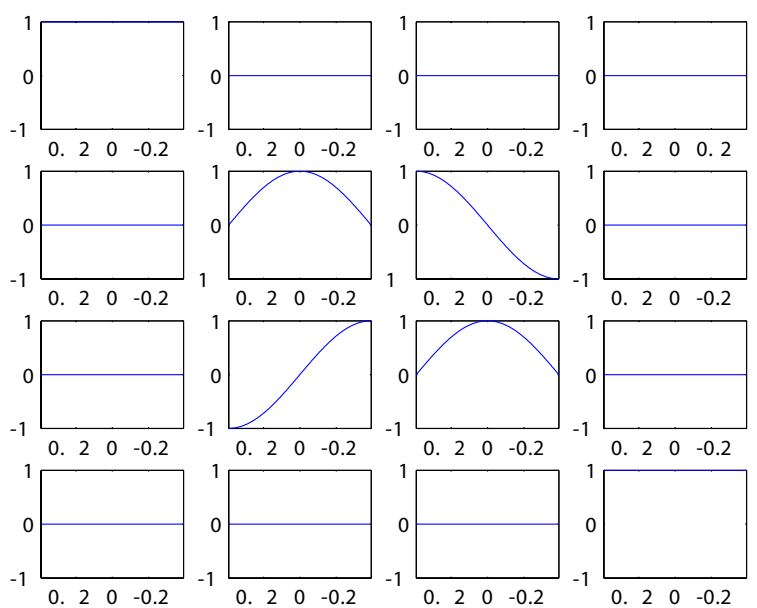

FIG. 5 Mueller matrix of an ideal half-wave plate rotating from $22.5^{\circ}$ downto $-22.5^{\circ}$.

tarder, prevents us from correctly determining $S_{2}$ component. This phenomenon can be alleviated by placing an additional quarter wave plate with a $0^{\circ}$-oriented fast axis in front of the cube. In this case, a large variation of $m_{23}$ is obtained versus gray level control, whereas $m_{24}$ remains small (Figure 6).

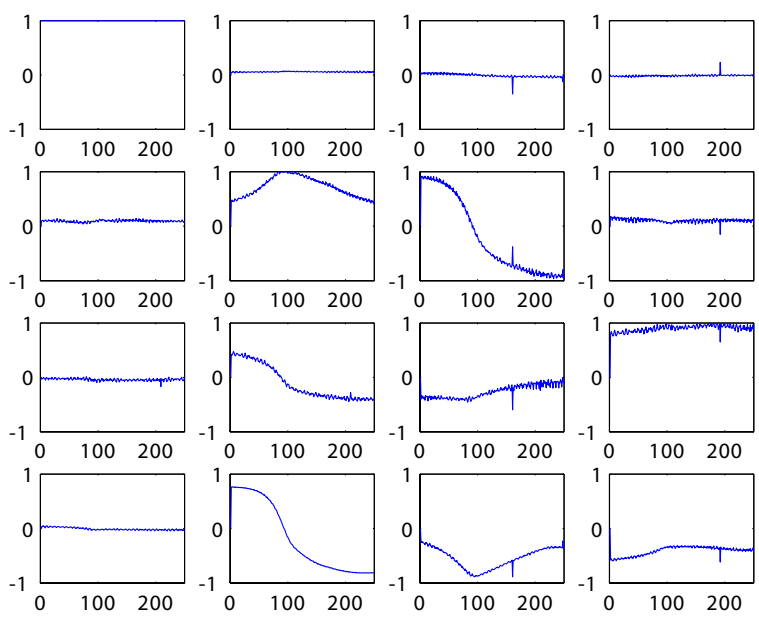

FIG. 6 Mueller matrix of the setup including the additional quarter-wave plate in front of the beamsplitter, the exit polarizer was not considered. Each Mueller matrix coefficient is plotted versus grey level control of the modulator.

In the polarimetric experiment described below, the FLC device is used in conjunction with a vertical polarizer placed in front of the camera. Therefore, only the first row of the Mueller matrix of our full system (ie the combination of the beamsplitter cube, the FLC device and the polarizer) is of interest (Figure 7). The coefficients in this row are compared with those of the combination of a half-wave plate and polarizer (Figure 8). The full optical setup should be able to be considered as a halfwave plate whose fast angle axis is tunable.

According to figures reported in Figure 7, the different grey levels we choose for partial Stokes vector analysis are levels 0, 92 and 255. Choice of levels 0 and 255 corresponds to extreme polarization directions; moreover, the two levels are generated in a single control thanks the modulator driver producing a DC-balanced control. Level 92 allows us to almost

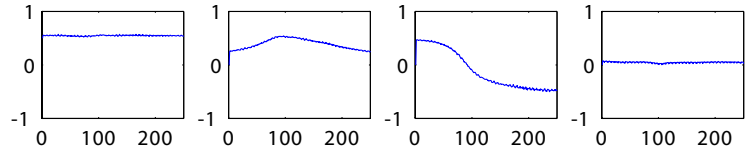

FIG. 7 Evolution vs. grey level of the coefficients in the first row of the Mueller matrix of the combination of the FLC device and polarizer.
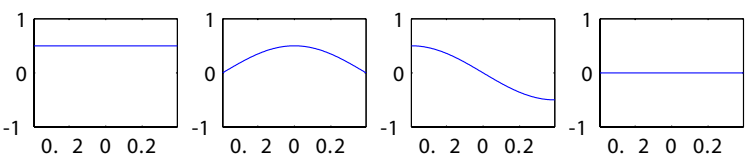

FIG. 8 Evolution vs. angle of the coefficients in the first row of Mueller matrix of the combination of a rotating half-wave plate and a polarizer (model).

eliminate Mueller coefficients $m_{11}$ and $m_{12}$. Relation between intensities and Stokes parameters is then a simplified analysis matrix with rank 3 obtained by Mueller matrix of PSA $\left(M_{P S A}\right)$ composed with a quarter-wave plate, the FLC modulator and a polarizer:

$$
\begin{gathered}
A_{r}=\left[\begin{array}{ccc}
\left(M_{P S A(0)}\right)_{11} & \left(M_{P S A(0)}\right)_{12} & \left(M_{P S A(0)}\right)_{13} \\
\left(M_{P S A(92)}\right)_{11} & \left(M_{P S A(92)}\right)_{12} & \left(M_{P S A(92)}\right)_{13} \\
\left(M_{P S A(255)}\right)_{11} & \left(M_{P S A(255)}\right)_{12} & \left(M_{P S A(255)}\right)_{13}
\end{array}\right] \\
A_{r}=\left[\begin{array}{lll}
0.548 & 0.249 & 0.463 \\
0.548 & 0.537 & 0.004 \\
0.556 & 0.242 & -0.454
\end{array}\right]
\end{gathered}
$$

In this configuration, identifying to Eq. (13), the FLC device is equivalent to a half-wave plate with angles of $-15^{\circ}$ (grey level 0 ), $0^{\circ}$ (grey level 92 ), $15^{\circ}$ (255 or opposite of grey level 0 ). We can evaluate the incident Stokes vector, or at least, its first three components, using $A_{r}^{+}$, which in this case (three measurements) comes down to $A_{r}^{-1}$.

$$
\left[\begin{array}{l}
S_{0} \\
S_{1} \\
S_{2}
\end{array}\right]=A_{r}^{+} \cdot\left[\begin{array}{c}
I_{0} \\
I_{92} \\
I_{255}
\end{array}\right]=A_{r}^{-1} \cdot\left[\begin{array}{c}
I_{0} \\
I_{92} \\
I_{255}
\end{array}\right]
$$

with

$$
A_{r}^{-1}=\left[\begin{array}{ccc}
1.649 & -1.516 & 1.668 \\
-1.691 & 3.410 & -1.694 \\
1.118 & -0.039 & -1.063
\end{array}\right]
$$

\section{EXPERIMENTAL VALIDATION AND RESULTS}

Two optical setups were implemented to evaluate partial Stokes parameters: one considers transmissive samples (Section 4.1) and the other one reflective samples (Section 4.2). These setups are used at an acquisition frequency of $234 \mathrm{~Hz}$. 
The FLC modulator is driven using four images, two with respective grey levels of 0 and 92 and their opposite images. The opposite image of level 92 image is not actually used for the evaluation of the Stokes parameters.

In both setups, samples are illuminated with a linearly polarized He-Ne beam at $632.8 \mathrm{~nm}$ placed behind a rotating diffuser. This diffuser exhibits a depolarization of $2 \%$. The scene, either transmissive or reflective, is imaged onto the SLM which is imaged onto the camera through a polarizer. The camera works at a fixed data transmission bandwidth, therefore its resolution has been reduced to $648 \times 80$ in order to increase its speed up to $234 \mathrm{~Hz}$.

\subsection{Experimental validation with a transmissive scene}

\subsubsection{Transmissive configuration description}

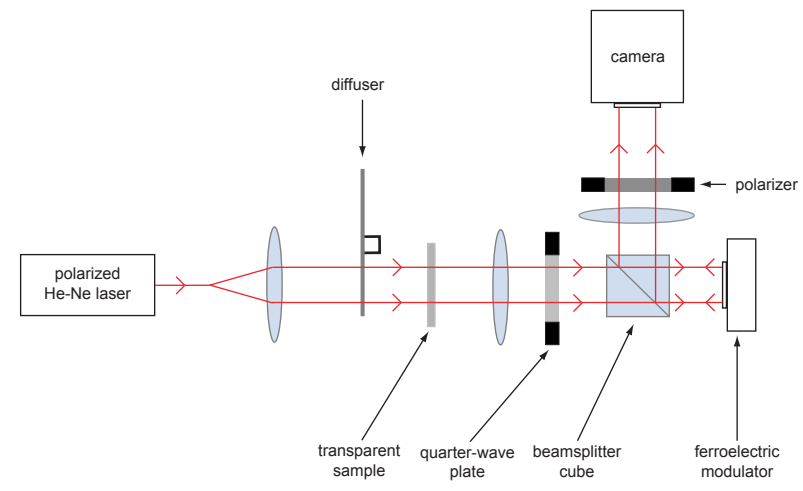

FIG. 9 Optical setup for the transmissive configuration.

The transmissive setup is described (Figure 9). We used a scene depicted in Figure 10, composed of 3 poor-quality polarizers whose orientations are different: approximately vertical, horizontal and with $45^{\circ}$ angle. Polarization of the incident beam is circular, which results in 4 basic polarizations after the scene (vertical, horizontal, at $45^{\circ}$ angle and circular).

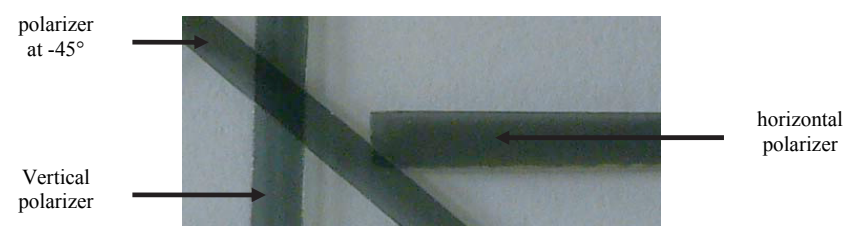

FIG. 10 Scene with 3 polarizers having different polarization orientation.

\subsubsection{Static results in transmission}

We compared the performance of our dynamic setup with that of a static setup composed of a rotating half-wave plate. Two comparisons were performed. The first one considers a normalized partial Stokes vector (Figure 11): $S_{0}$, normalized $S_{1}$ $\left(S_{1} / S_{0}\right)$, normalized $S_{2}\left(S_{2} / S_{0}\right)$.

We then considered $D O P_{L}$ and the angle of linear polarization $\Psi$ (Figure 12). From a qualitative point of view, these images are quite satisfactory; the three polarizers are clearly different.

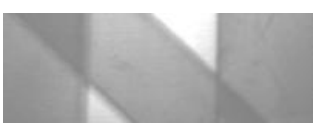

(a)

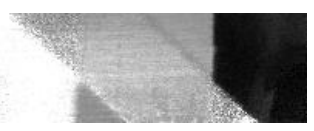

(c)

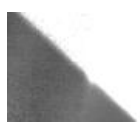

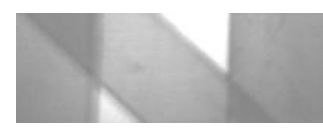

(b)

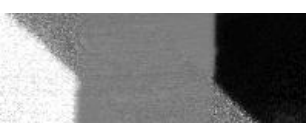

(d)

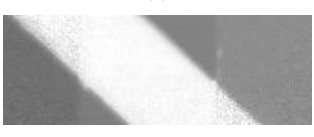

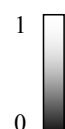
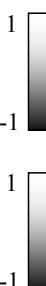

FIG. 11 Comparison of experimental results obtained with two setups: with the SLM (a, $c$ and e) and with a half-wave plate (b, $d$ and f). We depict Stokes parameters $S_{0}$ (a and b), normalized $S_{1}\left(S_{1} / S_{0}\right)$ (c and d) and normalized $S_{2}\left(S_{2} / S_{0}\right)$ (e and f).

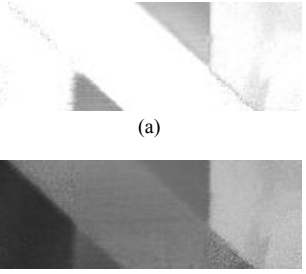

(c)
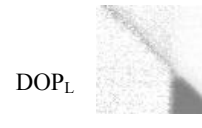

(b)

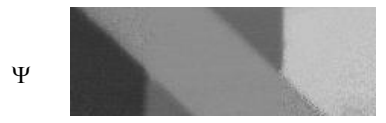

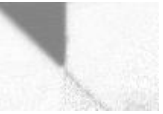
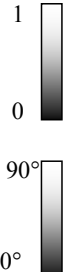

FIG. 12 Comparison of results obtained with two setups: with the SLM (a and c) and with a half-wave plate ( $b$ and $d)$, for the $D O P_{L}$ ( $a$ and $b$ ) and for the polarization angle ( $c$ and $d)$.

We report in Table 2 results obtained either with the FLC modulator or with the rotating half-wave. They prove rather similar. The figures have been evaluated over $10 \times 10$-pixel zones. These figures clearly report phenomena already noticeable in Figure 11, for instance a difference between both experiments as far as $S_{1}$ of the $+45^{\circ}$ polarizer is considered. They also show that the SLM-based experiments are less reliable than the experiments performed with rotating wave plate: $D O P_{L}$ figures strongly vary with the SLM-based polarimeter. We can explain it by noise and an inaccurate synthesis matrix $A^{+}$, since the determination of this latter is based on experimental data.

\subsubsection{Dynamic results in transmission}

In this Subsection, the conditions are similar to those in the previous sub-Section, except that the scene comes and goes in the horizontal direction during the acquisition. The corresponding speed ranges from $-0.07 \mathrm{mms}^{-1}$ to $0.07 \mathrm{mms}^{-1}$, which represents a maximal shift of 11.7 pixels between successive frames. High-quality images were obtained for Stokes parameters (Figure 13), imaging $D O P_{L}$ and angle of polarization (Figure 14). Some defaults can nevertheless be observed at the edge of objects because of translation. In this case, correction algorithms could be applied, provided that the displacement between consecutive acquisitions remains small $[31,32]$. 


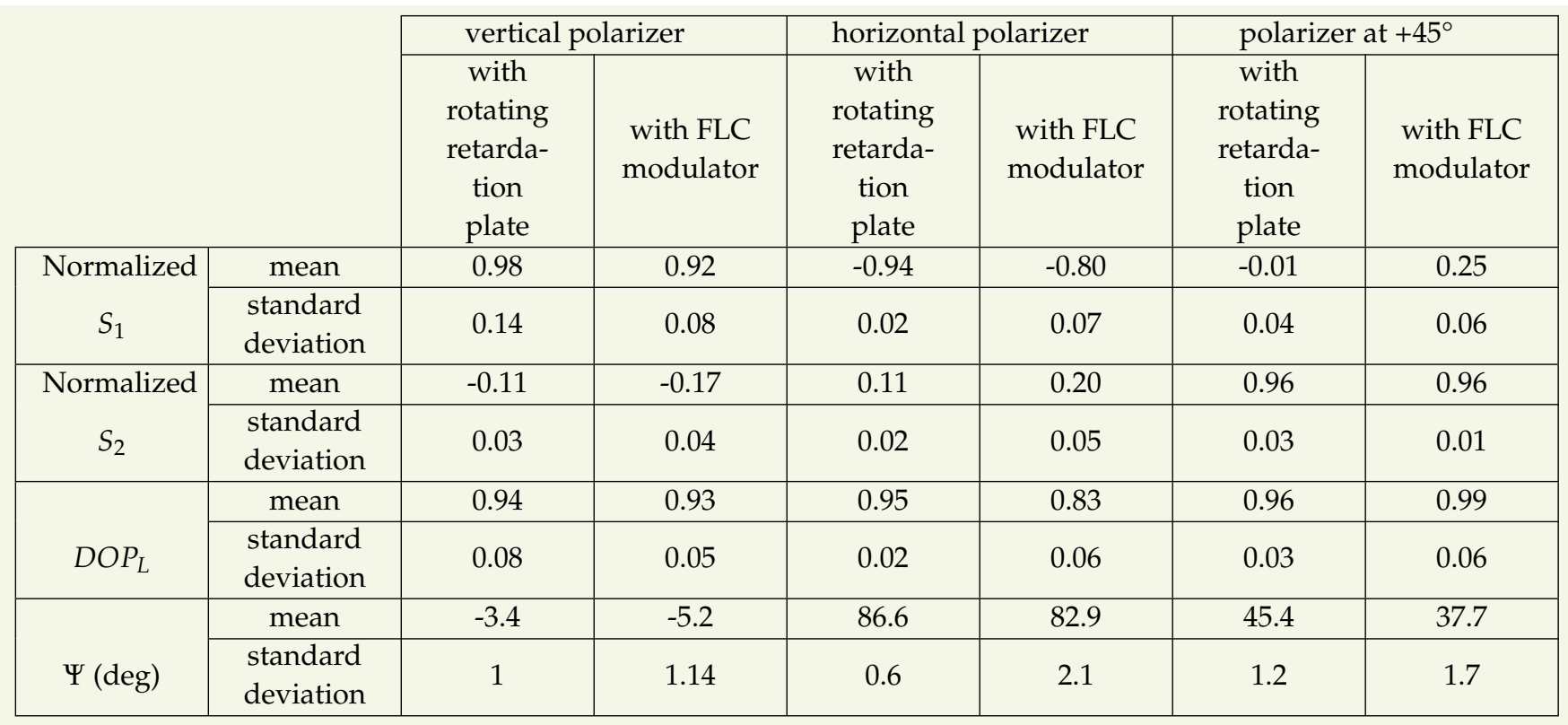

TABLE 2 Comparison of values obtained with the SLM-based setup and the rotating wave plate setup. We consider figures of the partial Stokes vector, $D O P_{L}$ and $\Psi$.

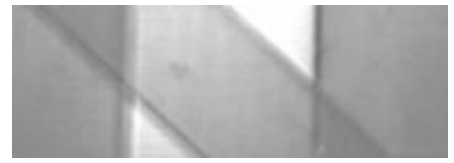

(a)

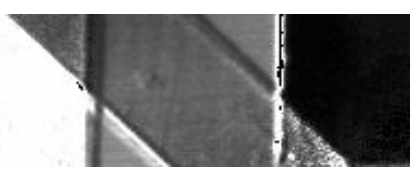

(b)

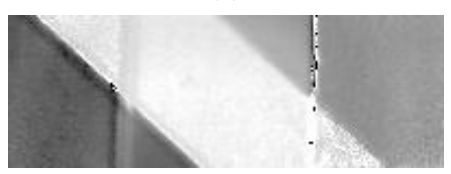

(c)

FIG. 13 Polarization images of samples obtained with SLM-based polarimeter. We report Stokes images $S_{0}(\mathrm{a}), S_{1}$ (b) and $S_{2}$ (c) in transmissive configuration. Contrary to results reported in Figure 11 and Figure 12, the samples are moving.

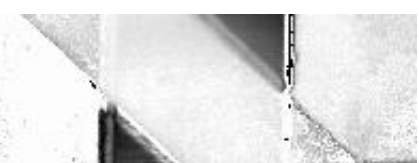

(a)

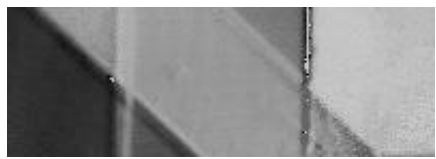

(b)
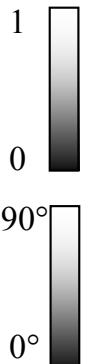

FIG. $14 D O P_{L}$ and polarization angle $\Psi$ images of samples obtained with SLM-based polarimeter for the scene in transmissive configuration.

\subsection{Experimental validation with a reflective scene}

\subsubsection{Reflective configuration description}

The reflective setup is described (Figure 15). In order to prove the ability of our device to capture dynamic phenomena, we used a rotating scene (Figure 16). It is composed of an aluminium plate on which several objects are placed: a polystyrene chip, a one euro cent coin which is fixed with a piece of double-sided adhesive tape and several layers of translucent adhesive tape which have been superimposed.

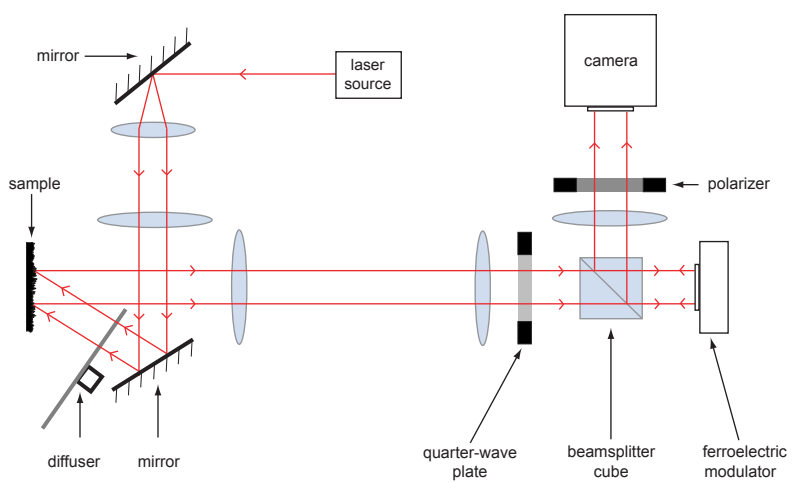

FIG. 15 Optical setup for the reflective configuration.

\subsubsection{Dynamic results in reflection}

These elements modify polarization in various ways, but in this study we are only interested in evaluating depolarization. For instance, we will not consider the translucent adhesive tape which exhibits birefringence in addition to depolarization. We first observed the polystyrene chip, the coin and the double sided adhesive tape.

The scene has been illuminated by a linear polarized laser with polarization axis of $45^{\circ}$. Despite noise, quality images have been obtained for the first three Stokes parameters (Fig- 


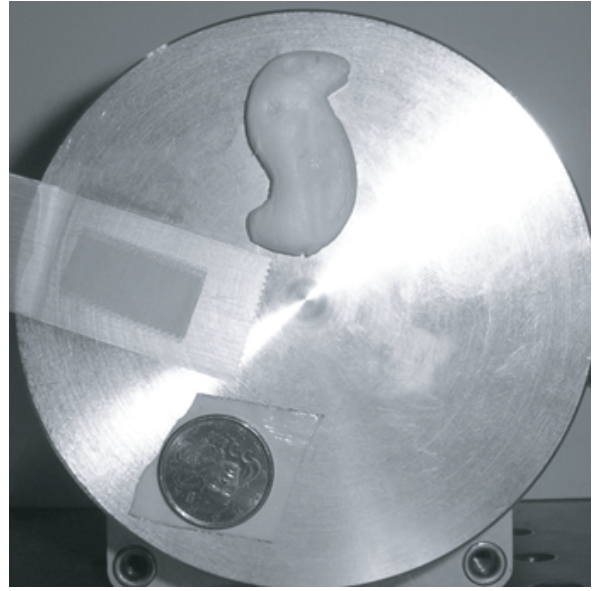

FIG. 16 Rotating scene used in our dynamic test in reflection.

ure 17) and $D O P_{L}$ (Figure 18). Polarization properties have been observed for these images. The coin and the aluminium background do not depolarize, but the double sided adhesive tape and polystyrene chip depolarize strongly.

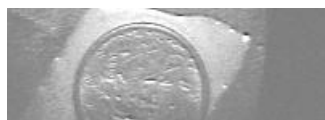

(a)

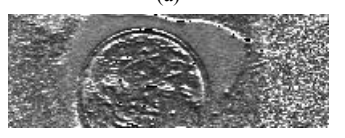

(c)

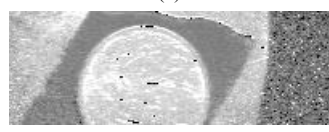

(e)

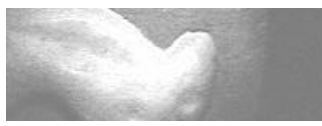

(b)
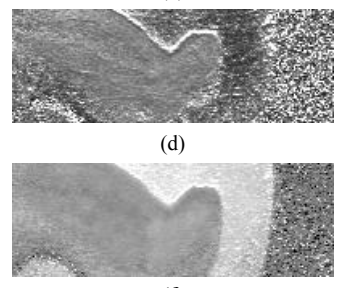

(f)

FIG. 17 Polarization images obtained with the SLM : Stokes parameters $S_{0}(\mathrm{a}, \mathrm{b})$, normalized $S_{1}(\mathrm{c}, \mathrm{d})$ and normalized $S_{2}(\mathrm{e}, \mathrm{f})$ in reflective configuration.

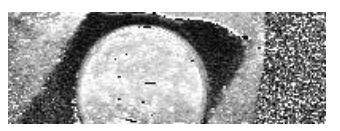

(a)

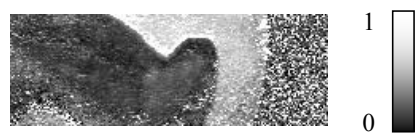

(b)

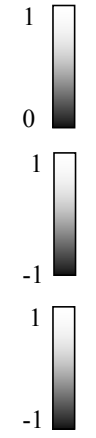

FIG. $18 D O P_{L}$ images of samples obtained with the SLM for the scene in transmissive configuration.

We can compare normalized $S_{1}$, normalized $S_{2}$ and $D O P_{L}$ images reported in Figure 17 with those obtained with the rotating quarter wave plate polarimeter (Table 3). These figures are averaged over $10 \times 10$-pixel uniform zones. The results prove quite similar, except the DOP of the double sided adhesive tape which seems to be overevaluated in the case of the dynamic polarimeter. Actually, major differences are noticeable in highly depolarizing zones. Like for previous experiments when we considered the three polarizers, variations between SLM-based experiments and wave plate-based experiments can be explained by noise and an inaccurate $A^{+}$determination. For non depolarizing objects, figures relative to $S_{2}$ are much larger than those relative to $S_{1}$ since the incident Stokes vector is $(1,0,+1,0)$, ie aligned at $45^{\circ}$.

\section{DISCUSSION}

Previous results, obtained either with a static or a dynamic scene, clearly prove that using a single ferroelectric liquid crystal modulator for high speed partial Stokes vector evaluation may be an adequate solution. The first three Stokes components and then $D O P_{L}$ and polarization angle can be evaluated at $234 \mathrm{~Hz}$.

Nevertheless, this implementation has several drawbacks: a weak point is clearly the FLC modulator which exhibits, like other silicon backplane devices, an overall spatial uniformity issue due to a non planar mirror $[33,34]$ and also local nonuniformity issues.

Figure 19 describes the resulting imaging analysis matrix. Clearly, only the central part of the modulator can be trusted, which can explain some inaccurate results. An improvement could be to use this imaging analysis matrix instead of the averaged matrix we used, but this would result in either a slower or a more complex computer implementation.
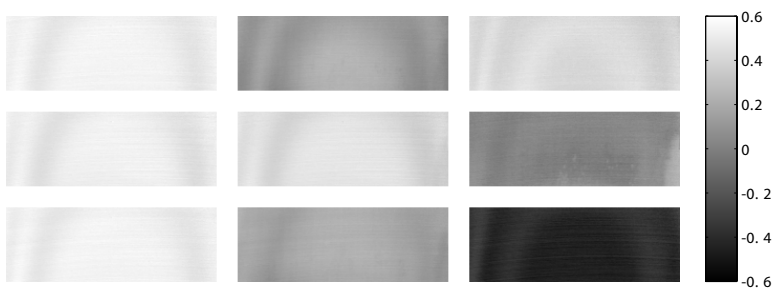

FIG. 19 Imaging analysis matrix of the full optical setup.

The use of a transmissive modulator may be a solution, but to our knowledge, presently, only bistable transmissive modulators were reported in such implementations [35]. This implementation could also be enhanced with the use of a faster camera, since the modulator is only used at $234 \mathrm{~Hz}$, whereas it can reach up to $1015 \mathrm{~Hz}$. Actually, for a possible evaluation of the first three Stokes coefficients, there is no need for the modulator to be similar to a tunable half-wave plate. The only condition is an analysis matrix with a rank higher or equal to three, which is the case with nematic liquid crystal modulators for instance, but these latter definitely operate too slowly. Nevertheless, there is a unique advantage in using a half-wave plate or similar: in this case, intensities images that are acquired can be easily interpreted and may be more closely related to physical characteristics of the objects, which can be useful when image post-processing, like segmentation, is considered [36].

\section{CONCLUSION}

We successfully implemented a high speed imaging polarimeter using a single ferroelectric liquid crystal light modulator. This setup is simpler than similar devices which were previously reported. It provides partial Stokes information (hence linear degree of polarization and angle of polarization) at 234 


\begin{tabular}{|c|c|c|c|c|c|c|c|c|}
\hline & \multicolumn{2}{|c|}{$\begin{array}{c}\text { Values obtained for } \\
\text { double sided adhesive } \\
\text { tape }\end{array}$} & \multicolumn{2}{|c|}{$\begin{array}{c}\text { Values obtained for } \\
\text { euro cent coin }\end{array}$} & \multicolumn{2}{c|}{$\begin{array}{c}\text { Values obtained for } \\
\text { aluminium wheel }\end{array}$} & \multicolumn{2}{c|}{$\begin{array}{c}\text { Values obtained for } \\
\text { polystyrene chip }\end{array}$} \\
\cline { 2 - 8 } & $\begin{array}{c}\text { with } \\
\text { rotating } \\
\text { retarda- } \\
\text { tion } \\
\text { plate }\end{array}$ & $\begin{array}{c}\text { with FLC } \\
\text { modula- } \\
\text { tor }\end{array}$ & $\begin{array}{c}\text { with } \\
\text { rotating } \\
\text { retarda- } \\
\text { tion } \\
\text { plate }\end{array}$ & $\begin{array}{c}\text { with FLC } \\
\text { modula- } \\
\text { tor }\end{array}$ & $\begin{array}{c}\text { with } \\
\text { rotating } \\
\text { retarda- } \\
\text { tion } \\
\text { plate }\end{array}$ & $\begin{array}{c}\text { with FLC } \\
\text { modula- } \\
\text { tor } \\
\text { rotating } \\
\text { retarda- } \\
\text { tion } \\
\text { plate }\end{array}$ & $\begin{array}{c}\text { with FLC } \\
\text { modula- } \\
\text { tor }\end{array}$ \\
\hline $\begin{array}{c}\text { Normal- } \\
\text { ized } S_{1}\end{array}$ & 0.02 & 0.05 & 0.07 & 0.08 & 0.03 & 0.04 & 0.06 & 0.09 \\
\hline $\begin{array}{c}\text { Normal- } \\
\text { ized } S_{2}\end{array}$ & 0.02 & 0.11 & 0.81 & 0.80 & 0.84 & 0.86 & 0.14 & 0.17 \\
\hline $\begin{array}{c}\text { Normal- } \\
\text { ized } \\
\text { DOP }\end{array}$ & 0.03 & 0.12 & 0.81 & 0.80 & 0.84 & 0.86 & 0.16 \\
\hline
\end{tabular}

TABLE 3 Comparison between normalized $S_{1}$, normalized $S_{2} D O P_{l}$ and $\Psi$ obtained with the rotating retardation plate polarimeter and with the FLC polarimeter for different samples of our dynamic test rotating reflective scene.

$\mathrm{Hz}$, much faster than polarimeters which use nematic liquid crystal light modulators. This setup is able to capture moving scenes, providing quality images. A possible improvement to this setup may be to use a higher quality modulator and a faster camera.

\section{ACKNOWLEDGEMENTS}

We thank the anonymous reviewers who carefully read the original version and wrote very accurate and detailed comments that helped us in improving the manuscript.

\section{References}

[1] J.M. Bueno and P. Artal, "Double-pass imaging polarimetry in the human eye" Opt. Lett. 24, 64-66 (1999).

[2] B. Laude-Boulesteix, A. de Martino, B. Drévillon, and L. Schwartz, "Mueller Polarimetric Imaging System with Liquid Crystals" Appl. Optics 43, 2824-2832 (2004).

[3] P.-Y. Gerligand, R. A. Chipman, E. A. Sornsin, and M. H. Smith, "Polarization signatures of spherical and conical targets measured by Mueller matrix imaging polarimetry" in Polarization: Measurement, Analysis, and Remote Sensing D. H. Goldstein and R. A. Chipman, eds. 3121, 63-73 (Proc. SPIE, San Diego, USA 1997).

[4] G. D. Lewis, D. L. Jordan, and P. J. Roberts, "Backscattering Target Detection in a Turbid Medium by Polarization Discrimination" Appl. Optics 38, 3937-3944 (1999).

[5] B. J. Deboo, J. M. Sasian, and R. A. Chipman, "Depolarization of diffusely reflecting man-made objects" Appl. Optics 44, 5434-5445 (2005).

[6] M. Floc'h, G. Le Brun, J. Cariou, and J. Lotrian, “Experimental characterization of immersed targets by polar decomposition of the Mueller matrices" Eur Phys J-Appl Phys 3, 349-358 (1998).

[7] G. W. Kattawar and M. J. Rakovic, "Virtues of Mueller Matrix Imaging for Underwater Target Detection" Appl Optics 38, 6431-6438 (1999).

[8] M. Alouini, D. Dolfi, F. Goudail, and P. Réfrégier, “Detection enhancement of low contrast targets through active multispectral polarimetric imaging" in Advanced Imaging Techniques, 10-12 (EOS Topical Meeting on Advanced Imaging Techniques, Delft, 2003).

[9] F. A. Sadjadi, “Passive three-dimensional imaging using polarimetric diversity" Opt. Lett. 32, 229-231 (2007).

[10] 0. Morel, C. Stolz, F. Mériaudeau, and P. Gorria, “Active Lighting Applied to 3D Reconstruction of Specular Metallic Surfaces by Polarization Imaging" Appl. Optics 45, 4062-4068 (2006).

[11] J.-Y. Lin, K.-H. Chen, and J.-H. Chen, "Optical method for measuring optical rotation angle and refractive index of chiral solution" Appl. Optics 46, 8134-8139 (2007).

[12] R. Hegedus, S. Akesson, and G. Horvath, "Polarization patterns of thick clouds: overcast skies have distribution of the angle of polarization similar to that of clear skies" J. Opt. Soc. Am. A 24, 2347-2356 (2007).

[13] D. Goldstein, Polarized light second edition (Marcel Dekker, NewYork Basel, 2003).

[14] F. A. Sadjadi and C. S. L. Chun, "Remote sensing using passive infrared Stokes parameters" Opt. Eng. 43, 2283-2291 (2004).

[15] L. B. Wolff, T. A. Mancini, P. Pouliquen, and A. G. Andreou, "Liquid crystal polarization camera" IEEE T. Robotic. Autom. 13, 195-203 (1997).

[16] Y. Hanaoka, "Ferroelectric Liquid Crystal Polarimeter for Highcadence Halpha Imaging Polarimetry" Solar Physics 222, 265-278 (2004).

[17] R. A. Chipman, "Depolarization index and the average degree of polarization" Appl. Optics 44, 2490-2495 (2005).

[18] M. Alouini, F. Goudail, P. Refregier, A. Grisard, E. Lallier, and D. Dolfi, "Multispectral polarimetric imaging with coherent illumination: towards higher image contrast" in Polarization: Measurement, Analysis, and Remote Sensing VI, D. H. Goldstein and D. B. Chenault, eds. 5432, 133-144 (Proc. SPIE, Orlando, USA, 2004).

[19] M. W. Williams, "Depolarization and cross polarization in ellipsometry of rough surfaces" Appl. Optics 25, 3616-3622 (1986).

[20] P. Clémenceau, A. Dogariu, and J. Stryewski, "Polarization active imaging" in Laser Radar Technology and Applications V, G. W. Kamerman, U. N. Singh, C. Werner, and V. V. Molebny, eds. 4035, 401-409 (Proc. SPIE, Orlando, USA, 2000).

[21] J. S. Tyo, "Design of optimal polarimeters: maximization of signalto-noise ratio and minimization of systematic error" Appl. Optics 
41, 619-630 (2002).

[22] F. Goudail, P. Terrier, Y. Takakura, L. Bigué, F. Galland, and V. Devlaminck, "Target detection with a Liquid Crystal-based passive Stokes polarimeter" Appl. Optics 43, 274-282 (2004).

[23] Meadowlark-0ptics, "Stokes Polarimetry Using Liquid-Crystal Variable Retarders" (2001), http://www.meadowlark.com.

[24] A. M. Gandorfer, "Ferroelectric retarders as an alternative to piezoelastic modulators for use in solar Stokes vector polarimetry" Opt. Eng. 38, 1402-1408 (1999).

[25] K. A. Bauchert, S. A. Serati, and A. Furman, "Advances in liquid crystal spatial light modulators" in Optical Pattern Recognition XIII, D. P. Casasent and T.-H. Chao, eds. 4734, 35-43 (Proc. SPIE, Orlando, USA, 2002)

[26] S. A. Serati, G. D. Sharp, R. A. Serati, D. J. McKnight, and J. E. Stockley, "128 $\times 128$ analog liquid crystal spatial light modulator" in Optical Pattern Recognition VI, D. P. Casasent and T.-H. Chao, eds. 2490, 378-387 (Proc. SPIE, Orlando, USA, 1995).

[27] K. A. Bauchert and S. A. Serati, "High-speed multi-level $512 \times 512$ spatial light modulator" in Optical Pattern Recognition XI, D. P. Casasent and T.-H. Chao, eds. 4043, 59-65 (Proc. SPIE, Orlando, USA, 2000).

[28] Boulder-Nonlinear-Systems, “Liquid crystal spatial light modulator: 512 × 512 multi-level/analog" (2002), http://www.bnonlinear.com/productframeset.html.

[29] L. Bigué, L. Jourdainne, and P. Ambs, "High speed ferroelectric gray-scale spatial light modulator for implementing diffractive optical elements" in Diffractive Optics and Micro-Optics, R. Magnus- son, ed. TOPS 75, 58-62 (OSA, Tucson, USA, 2002).

[30] A. Jaulin, L. Bigué, and P. Ambs, “High-speed degree of polarization imaging with a ferroelectric liquid crystal modulator" Opt. Eng. 47, 033201 (2008).

[31] S. Guyot, M. Anastasiadou, E. Deléchelle, and A. de Martino, "Registration scheme suitable to Mueller matrix imaging for biomedical applications" Opt. Express 15, 7393-7400 (2007).

[32] L. Gendre, A. Foulonneau, and L. Bigué, "Correction of erroneous degree of polarization of moving objects in a video sequence" in Polarization: Measurement, Analysis, and Remote Sensing VIII, D. B. Chenault and D. H. Goldstein, eds. 6972, 69720R (Proc. SPIE, Orlando, USA, 2008)

[33] J. L. Harriman, A. Linnenberger, and S. A. Serati, "Improving spatial light modulator performance through phase compensation" in Advanced Wavefront Control: Methods, Devices, and Applications II, J. D. Gonglewski, M. T. Gruneisen, M. K. Giles, eds. 5553, 58-67 (Proc. SPIE, Denver, USA, 2004).

[34] W. N. Hart, C. M. Roggemann, A. Sergeyev, and J. T. Schulz, "Characterizing static aberrations in liquid crystal spatial light modulators using phase retrieval" Opt. Eng. 46, 086601 (2007).

[35] L. Bigué and N. Cheney, "High-speed portable polarimeter using a ferroelectric liquid crystal modulator" in Polarization science and Remote Sensing III, J. A. Shaw and J. S. Tyo, eds., 6682, 668205 (Proc. SPIE, San Diego, USA, 2007).

[36] J.E. Ahmad, and Y. Takakura, "Improving Segmentation Maps using Polarization Imaging" in ICIP 2007 1, I-281-I-284 (IEEE, San Antonio, USA, 2007) 